\title{
Duality covariant type-IIB supersymmetry and nonperturbative consequences
}

\author{
Itzhak Bars \\ Theory Division, CERN, CH-1211 Geneva 23, Switzerland \\ and Department of Physics and Astronomy, University of Southern California, Los Angeles, California 90089-0484
}

(Received 27 June 1997)

\begin{abstract}
Type-IIB supersymmetric theories have an $\operatorname{SL}(2, Z)$ invariance, known as $U$ duality, which controls the nonperturbative behavior of the theory. Under $\operatorname{SL}(2, Z)$ the supercharges are doublets, implying that the bosonic charges would be singlets or triplets. However, among the bosonic charges there are doublet strings and doublet five-branes which are in conflict with the doublet property of the supercharges. It is shown that the conflict is resolved by structure constants that depend on moduli, such as the tau parameter, which transform under the same $\operatorname{SL}(2, Z)$. The resulting superalgebra encodes the nonperturbative duality properties of the theory and is valid for any value of the string coupling constant. The usefulness of the formalism is illustrated by applying it to purely algebraic computations of the tension of $(p, q)$ strings, and the mass and entropy of extremal black holes constructed from $D$-1-branes and $D$-5-branes. In the latter case the nonperturbative coupling dependence of the BPS mass and renormalization is computed for the first time in this paper. It is further argued that the moduli dependence of the superalgebra provides hints for four more dimensions beyond ten, such that the superalgebra is embedded in a fundamental theory which would be covariant under $\operatorname{SO}(11,3)$. An outline is given for a matrix theory in 14 dimensions that would be consistent with $M$ (atrix) theory as well as with the above observations. [S0556-2821(97)02122-X]
\end{abstract}

PACS number(s): 11.25.Hf, 04.50.+h, 04.70.Dy, 11.25.Mj

\section{EXTENDED TYPE-IIB SUPERSYMMETRY AND DUALITY}

Type-IIB supergravity and superstrings have two supercharges $Q_{\alpha a}=\left(Q_{\alpha(1)}, Q_{\alpha(2)}\right)$. The 16-component real spinors, $\alpha=1, \ldots, 16$, have the same ten-dimensional chirality. They are both the $\mathbf{1 6}$ of $\mathrm{SO}(9,1)$. This is to be contrasted with type-IIA supercharges that are one $\mathbf{1 6}$ and one $\overline{\mathbf{1 6}}$. In the perturbative formulation of superstrings, the transformation rules of the dynamical variables are such that, the 32 real supercharges obey the unextended superalgebra

$$
\left\{Q_{\alpha a}, Q_{\beta b}\right\}=\delta_{a b} \gamma_{\alpha \beta}^{\mu} P_{\mu},
$$

where $P_{\mu}$ is the momentum operator in ten dimensions, with $\mu=0,1, \ldots, 9$.

The discovery of duality symmetries led to a larger picture in which strings are in interaction with $D$ - $p$-branes [1]. ${ }^{1}$ In this case the superalgebra must have central extensions. The dynamical details of the underlying theory are still being developed. However, independent of dynamical details, there is a lot of information encoded in the superalgebra and we would like to take maximum advantage of this information. The fully generalized superalgebra of type-IIB in ten dimensions is

\footnotetext{
${ }^{1}$ An action of an open string interacting with zero branes (particles) at its end points was suggested and studied a long time ago with a different physical emphasis [2]. A reexamination of the approach in the context of modern ideas may be enlightening.
}

$$
\begin{aligned}
\left\{Q_{\alpha a}, Q_{\beta b}\right\}= & \gamma_{\alpha \beta}^{\mu}\left(p_{\mu} \delta_{a b}+e_{\mu} \tau_{a b}^{3}+d_{\mu} \tau_{a b}^{1}\right) \\
& +\gamma_{\alpha \beta}^{\mu_{1} \mu_{2} \cdots \mu_{5}}\left(\begin{array}{c}
x_{\mu_{1} \mu_{2} \cdots \mu_{5}} \delta_{a b} \\
+y_{\mu_{1} \mu_{2} \cdots \mu_{5}} \tau_{a b}^{3} \\
+z_{\mu_{1} \mu_{2} \cdots \mu_{5}} \tau_{a b}^{1}
\end{array}\right) \\
& +\gamma_{\alpha \beta}^{\mu_{1} \mu_{2} \mu_{3}} t_{\mu_{1} \mu_{2} \mu_{3}}\left(i \tau^{2}\right)_{a b},
\end{aligned}
$$

where $\tau^{1,2,3}$ are $2 \times 2$ Pauli matrices. This superalgebra is meant to apply to any form of the theory, including the string approximation, or the low energy approximation in the form of IIB supergravity with all of its solitonic $D$ - $p$-brane configurations. The extensions $\left(e_{\mu}, d_{\mu}\right)$ correspond to elementary strings and $D$-1-strings, respectively, $t_{\mu_{1} \mu_{2} \mu_{3}}$ corresponds to three-branes, and the self-dual tensors $x_{\mu_{1} \mu_{2} \cdots \mu_{5}}$, $y_{\mu_{1} \mu_{2} \cdots \mu_{5}}$, and $z_{\mu_{1} \mu_{2} \cdots \mu_{5}}$ correspond to three types of selfdual five-branes.

Altogether there are 528 bosonic operators, including the "momentum" $p_{\mu}$. Other odd $p$-branes $(p=-1,7,9)$ that can appear in type-IIB theory are related to the same set of 528 bosons after dualizing them. Note that we have deliberately used an uppercase $P_{\mu}$ in Eq. (1) and a lowercase $p_{\mu}$ in Eq. (2). This is because the general relation between the two involves moduli parameters in the nonperturbative theory, as will be explained in the next section. This is why we used in quotes "momentum" in referring to $p_{\mu}$. For the same reasons we should refer to $\left(e_{\mu}, d_{\mu}\right)$ as "strings" in quotes. The true momentum $P_{\mu}$ and strings $\left(E_{\mu}, D_{\mu}\right)$ are linear combinations of $\left(p_{\mu}, e_{\mu}, d_{\mu}\right)$.

We will find that, in the general theory of type IIB, the relation between the two sets involves six functions of moduli that are Lorentz invariant in ten dimensions (10D). One of the moduli is the string coupling constant $g_{s}$ related 
to the dilaton in the usual way. This will follow quite generally from the $\mathrm{SL}(2, Z)_{U} U$-duality properties of the type-IIB theory. It will be demonstrated that in certain computations involving Bogomol'nyi-Prasad-Sommerfield (BPS) sectors, only the usual complex modulus $\tau$ enters in all expressions. Along the way we will discover that the $\tau$ modulus together with at least one more real modulus is needed to achieve $\operatorname{SL}(2, Z)$ covariance of the superalgebra. We will show that this symmetry and the moduli can be interpreted as if there are four hidden dimensions with signature $(2,2)$ and that three (six) moduli can be constructed from one (two) $\mathrm{SO}(2,2)$ on-shell vector(s).

It is of interest in the present paper to determine the general dependence of the 528 type-IIB bosons on the string coupling $g_{s}$ and other moduli in any general theory that includes all $D$ - $p$-branes. Recall first the form of the superalgebra for elementary strings. In perturbative string theory, without the $D$ - $p$-branes, $g_{s} \rightarrow 0$, the only extensions in the superalgebra are the winding numbers of the string, $e_{\mu} \rightarrow E_{\mu}$. So for elementary strings, at $g_{s}=0$, the algebra has the form ${ }^{2}$

$$
\lim _{g_{s} \rightarrow 0}\left\{Q_{\alpha a}, Q_{\beta b}\right\}=\gamma_{\alpha \beta}^{\mu}\left(P_{\mu} \delta_{a b}+E_{\mu} \tau_{a b}^{3}\right)
$$

If an elementary five-brane or elementary three-brane is included in the free theory, then one may add the corresponding extension that appears in Eq. (2) at $g_{s}=0$. In this paper the free limit will be taken as a boundary condition at $g_{s}$ $=0$. As we have learned from past experience, if one constructs an interacting action, $g_{s} \neq 0$, based on the superalgebra in Eq. (1) (e.g., type-IIB supergravity or superstrings), then one finds out later that for nonzero coupling and other nonzero moduli, the theory has nonperturbative solitons in the form of $D$ - $p$-branes. When all $p$-branes are present the nonperturbative theory develops all the extensions in the superalgebra (2), but with coefficients that depend on $g_{s}$ and the other moduli. It is our purpose here to find the general form of the dependence on the moduli by taking advantage of the $\operatorname{SL}(2, Z)_{U} U$-duality properties of the type-IIB theory. Of course, the general superalgebra should be compatible with Eq. (2) but our interest is in determining the nonperturbative moduli dependence of the various terms. The form of the superalgebra that we will find at $g_{s} \neq 0$ through this reasoning is expected to be valid in any formulation of the theory that includes the $D$-p-branes in interaction with

\footnotetext{
${ }^{2}$ For an elementary string $P_{\mu} \pm E_{\mu}$ are the zero modes of the left and right movers. Usually $E_{\mu}$ is taken to be zero in uncompactified directions because of boundary conditions on closed or open strings. Its nonvanishing components along compactified dimensions are determined by the geometry of compactification. For example, if the ninth dimension is compactified into a circle of radius $R_{9}$, then $E_{9}=n R_{9}$, where $n$ is the quantized winding number. In the general interacting case the boundary conditions can change for open strings and $E_{\mu}$ can become nonzero in all directions [3]. Since we are aiming at covering all possibilities, we will formally allow all components of $E_{\mu}$. In can then be specialized to the physical situation under discussion. The same general remarks apply for all other elementary $p$-branes.
}

strings, and thus encodes a certain amount of nonperturbative information independent of detailed dynamics.

It will be important to note the maximal symmetries of the general type-IIB superalgebra (2). It was first pointed out in [4] that in addition to $10 \mathrm{D}$ Lorentz symmetry $\mathrm{SO}(9,1)$, there is an $\operatorname{SL}(2, R)_{B}$ symmetry in the type-IIB superalgebra (2), including all extensions. Since the two supercharges are real, they naturally form a doublet $Q_{\alpha a}=\left(Q_{\alpha 1}, Q_{\alpha 2}\right)$ under an $\operatorname{SL}(2, R)_{B}$ transformation on the index $a=1,2$. In the symmetric product of the doublet there is a triplet and in the antisymmetric product there is a singlet of $\operatorname{SL}(2, R)_{B}$. Hence the superalgebra is written covariantly as

$$
\begin{aligned}
\left\{Q_{\alpha a}, Q_{\beta b}\right\}= & \left(i \tau_{2} \tau_{i}\right)_{a b}\left(\gamma_{\alpha \beta}^{\mu} p_{\mu}^{i}+\gamma_{\alpha \beta}^{\mu_{1} \mu_{2} \cdots \mu_{5}} x_{\mu_{1} \mu_{2} \cdots \mu_{5}}^{i}\right) \\
& +\left(i \tau_{2}\right)_{a b} \gamma_{\alpha \beta}^{\mu_{1} \mu_{2} \mu_{3}} t_{\mu_{1} \mu_{2} \mu_{3}},
\end{aligned}
$$

where the triplet is associated with the adjoint representation of $\operatorname{SL}(2, R)_{B}$, with Pauli matrices $\tau_{i} \equiv\left(-i \tau_{2}, \tau_{1},-\tau_{3}\right)$. These are related to the symmetric matrices $i \tau_{2} \tau_{i}$ $=\left(1, \tau_{3}, \tau_{1}\right)$. Hence the "momentum" and "strings" are members of the $\mathrm{SL}(2, R)_{B}$ triplet

$$
p_{\mu}^{i}=\left(p_{\mu}, e_{\mu}, d_{\mu}\right) .
$$

The self-dual five-branes also form a similar triplet, while the three-brane is a singlet.

The specialized form in Eq. (1) is invariant under the subgroup $\mathrm{SO}(2) \subset \mathrm{SL}(2, R)_{B}$. The one in Eq. (3) is invariant under the even smaller discrete subgroup $Z_{2} \subset \operatorname{SO}(2) \subset \operatorname{SL}(2, R)_{B}$ if any component of $E_{\mu}$ is nonzero (and transforms as $E_{\mu} \rightarrow-E_{\mu}$ under the $Z_{2}$ ). On the other hand, if the $D$-1-brane charge is turned on, the full triplet $p_{\mu}^{i}$ is available to provide a basis for the full $\mathrm{SL}(2, R)_{B}$ as in Eq. (4).

This symmetry has not received much attention so far. Is $\operatorname{SL}(2, R)_{B}$ related in any way to the $\operatorname{SL}(2, Z)_{U}$ of $U$ duality [5]? At first sight there seems to be no relation, because under $U$ duality the momentum $P_{\mu}$ is a singlet, while for $\operatorname{SL}(2, R)_{B}$ the "momentum" $p_{\mu}$ is a member of a triplet. Similarly, the elementary and $D$-1-strings are $\operatorname{SL}(2, Z)_{U}$ doublets, while the "strings" that appear above are members of a $\operatorname{SL}(2, R)_{B}$ triplet. This poses a puzzle as to what is the significance of the explicit $\operatorname{SL}(2, R)_{B}$ that stares at us in the superalgebra? We will see later that by introducing moduli that transform under both $\mathrm{SL}(2, Z)_{U}$ and $\mathrm{SL}(2, R)_{B}$ the connection between the two will be made.

From another point of view $\operatorname{SL}(2, R)_{B}$ has been connected to higher dimensions. In $S$ theory [4] three extra dimensions beyond 10 were associated with $\operatorname{SL}(2, R)_{B}=\mathrm{SO}(2,1)$ (spacelike 11th and 13th, and timelike 12th), but more recently in [6] it was understood that $\mathrm{SL}(2, R)_{B}$ is more properly associated with one of the $\operatorname{SL}(2, R)$ 's embedded in $\mathrm{SO}(2,2)=\mathrm{SL}(2, R)_{B} \otimes \mathrm{SL}(2, R)_{B^{\prime}}$ where $(2,2)$ corresponds to two timelike and two spacelike extra dimensions beyond 10 (spacelike 11th and 13th, timelike 12th \& 14th). Indeed we will see that the $\operatorname{SL}(2, Z)_{U}$ that transforms the moduli is precisely the discrete part of the $\operatorname{SL}(2, R)_{B}$ embedded in $\mathrm{SO}(2,2)$, and that a full set of six Lorentz-invariant moduli, including the coupling constant, are related to two "onshell" vectors of $\mathrm{SO}(2,2)$. 
In most of the paper we will concentrate on the type-IIB sector, and study some nonperturbative properties of the general theory by taking advantage of the $\mathrm{SL}(2, R)_{B} \leftrightarrow \mathrm{SL}(2, Z)_{U}$ connection. We will construct the nonperturbative type-IIB superalgebra with arbitrary values of the coupling constant and other moduli, show that its nonperturbative duality properties under $\operatorname{SL}(2, Z)_{U}$ are related to its hidden dimensions $\mathrm{SO}(2,2)$, and apply the superalgebra to the derivation of nonperturbative results in order to exhibit the utility and power of the approach. In particular, we will compute in a few steps the tension of $(p, q)$ strings, and the mass spectrum and entropy of black holes constructed from $D$-1-branes and D-5-branes.

The $\tau$ modulus that appears in such formulas, together with others needed to achieve $\operatorname{SL}(2, Z)$ covariance, will be related to on-shell momentumlike vectors in four dimensions, beyond the usual ten dimensions. This provides hints that in type-IIB supersymmetric theories there is a hidden 14-dimensional structure. More generally, it was claimed in [6] that a unified theory that is at least ten-dimensional Lorentz covariant and is also covariant under type-IIA or typeIIB duality is naturally a 14-dimensional theory with signature $(11,3)$, with 64 real supercharges. In this larger picture a constraint due to a gauge invariance restricts the theory to live only in the short representation sectors of the 64 supercharges. This constraint is expressed as a 14D covariant BPS-like master equation [6]. Dualities are naturally explained as symmetries of the master equation. For example, it was discovered in $S$ theory that type-IIA or type-IIB T duality corresponds to flipping the 13th dimension with the 9th dimension [4]. ${ }^{3}$ More will be said on this bigger picture in the last section of the paper, where a general outline for a 14D matrix formulation that contains dynamics, and which is consistent with the $M$ (atrix) model of [7], will be presented.

Finally, we remark that the methods of this paper can be extended to $U$-duality covariant superalgebras in dimensions lower than 10 , in particular to $N=2$ in four dimensions, but this will not be pursued in the present paper.

\section{MODULI, SL(2,Z) AND 14D}

\section{A. Hints from supergravity}

The uniqueness of supergravity theories is established on the basis of local supersymmetry. Accordingly, any type-IIB supersymmetric theory in ten dimensions that contains the graviton has type-IIB supergravity as its low energy limit in the massless sector. The massless spectrum of the superstring contains the type-IIB supergravity supermultiplet consisting of the graviton $g_{\mu \nu}$, two gravitinos $\psi_{\mu \alpha}^{a}$, two 2-index antisymmetric tensors $B_{\mu \nu}^{a}$, one self-dual 4-index antisymmetric tensor $A_{\mu \nu \lambda \sigma}$, two fermions $\chi_{\alpha}^{a}$, and two scalars [8]. The two scalars, identified as the dilaton $\phi$ and the axion $a$, are

\footnotetext{
${ }^{3}$ This is easy to see this by ignoring the 14 th dimension as in [4]. When compactified to 9 dimensions the type-IIA superalgebra with all 528 extensions has an $\mathrm{SO}(2,1)_{A}$ acting on the dimensions (9th,11th,12th $)_{A}$, while the type-IIB superalgebra has the $\operatorname{SO}(2,1)_{B}$ acting on the dimensions (13th,11th,12th) ${ }_{B}$. The 13th-9th flip is $T$ duality that maps these two into each other.
}

combined into the complex scalar field $\tau=a+i e^{-\phi}$. In the interacting supergravity theory, which corresponds to the low energy limit of the interacting superstring theory, one notices that there is an $\operatorname{SL}(2, R)_{U}$ symmetry. Under SL $(2, R)_{U}$ the bosons transform as follows: $g_{\mu \nu}, A_{\mu \nu \lambda \sigma}$ are singlets, $B_{\mu \nu}^{a}$ form a doublet acting on the index $a=1,2$, and the scalars undergo the fractional nonlinear Möbius transformation

$$
\tau_{g}=\frac{a \tau+b}{c \tau+d}, \quad g=\left(\begin{array}{ll}
a & b \\
c & d
\end{array}\right) \in \operatorname{SL}(2, R)_{U} .
$$

This may be understood by taking the scalars to belong to the coset $\operatorname{SL}(2, R)_{U} / \mathrm{SO}(2)$. The fermions $\left(\psi_{\mu \alpha}^{1}, \psi_{\mu \alpha}^{2}\right),\left(\chi_{\alpha}^{1}, \chi_{\alpha}^{2}\right)$ transform linearly like doublets under an induced gauged compact subgroup $\mathrm{SO}(2) \subset \mathrm{SL}(2, R)$ :

$$
\left(\begin{array}{cc}
\cos (\Lambda) & \sin (\Lambda) \\
-\sin (\Lambda) & \cos (\Lambda)
\end{array}\right) \in \operatorname{SO}(2) .
$$

The local gauge parameter of the transformation $\Lambda(x)$ depends on the global parameters $g \in \operatorname{SL}(2, R)_{U}$ as well as on the local scalar fields $\tau(x)$. Thus, the fermions feel the full $\operatorname{SL}(2, R)_{U}$ transformation in the combination $\Lambda(g, \tau(x))$ that involves the scalar fields nonlinearly.

The fermions are in one-to-one correspondence with the supercharges $\left(Q_{\alpha}^{1}, Q_{\alpha}^{2}\right)$. Therefore the supercharges must also transform as a doublet under the global part of $\mathrm{SO}(2)$, with a constant parameter $\Lambda(g, \tau)$, where $\tau$, called the modulus, is the constant part of $\tau(x)$. This modulus is related to the string coupling constant $g_{s}$, and in lowest order perturbative regime it takes the form

$$
\tau=\frac{\theta}{2 \pi}+i \frac{4 \pi^{2}}{g_{s}^{2}} .
$$

It transforms precisely as in Eq. (6). Thus, the supercharges of supergravity "feel" the global SL $(2, R)_{U}$ in the form of an induced subgroup $\mathrm{SO}(2)$ that depends on the string coupling constant. This is precisely the $\mathrm{SO}(2)$ symmetry of the perturbative superalgebra (1) discussed in the previous section. Evidently this $\mathrm{SO}(2)_{U=B}$ subgroup is in the nonperturbative $\operatorname{SL}(2, R)_{B}$ as seen in the previous section:

$$
\mathrm{SO}(2)_{U}=\mathrm{SO}(2)_{B} \subset \mathrm{SL}(2, R)_{B} \text {. }
$$

This provides a hint for how to connect $\operatorname{SL}(2, R)_{U}$ and $\operatorname{SL}(2, R)_{B}$.

In nonperturbative supergravity nonperturbative solitonic configurations of the fields are permitted. To describe these field configurations one must add more moduli (constants) corresponding to the boundary behavior of the fields that are different in the perturbative versus the nonperturbative theory. Effectively one introduces additional degrees of freedom through the "back door," as compared to the perturbative theory. The effect on the superalgebra of the presence of the nonperturbative moduli is to turn on central extensions, and hence change the supersymmetry properties of the theory in the nonperturbative regimes. The theory is no longer the same, and the supersymmetry transformation rules of the effective degrees of freedom must be a "new supersymmetry" transformation rule, so that the closure includes 
the central extensions. The extra charges together with compactified components of the momentum form multiplets under the so-called $U$-duality transformations. Hence the momentum as well as the supercharges cannot be immune to the $\operatorname{SL}(2, R)_{U}$ transformations. This begins to seem like the $\mathrm{SL}(2, R)_{B} \sim \mathrm{SL}(2, R)_{U}$ symmetry. But there seems to be conflicts in the transformation properties under $\operatorname{SL}(2, R)_{B}$ versus $\mathrm{SL}(2, R)_{U}$ as described in the previous section. The resolution of these conflicts involves the moduli and their transformation properties as described below.

\section{B. Moduli-dependent superalgebra}

The discussion above indicates that the nonperturbative supercharges and their superalgebra depend generally on the moduli. This must be true since they transform with the $\mathrm{SO}(2)_{U=B}$ whose parameters depend on the moduli. One of the moduli is the $\tau$ modulus (8) and we will see that generally there are more. To emphasize this property we will write $Q_{\alpha a}(\kappa)$ where $\{\kappa\}$ is a set of moduli. Since there is already a dependence on the moduli, it is convenient to choose a new basis for the $Q_{\alpha a}(\kappa)$ such that the basis is related to the old one with moduli-dependent coefficients. The coefficients can be taken as functions of the coset $\mathrm{SL}(2, R) / \mathrm{SO}(2)$ such that the $Q_{\alpha a}(\kappa)$ transform linearly under $\operatorname{SL}(2, R)$ $=\mathrm{SL}(2, R)_{U=B}$. That is, the modulus-dependent $\mathrm{SO}(2)$ transformation is compensated by the coefficients so that $Q_{\alpha a}(\kappa)$ transforms with modulus-independent $g \in \mathrm{SL}(2, R)$. One can go back and forth between the two bases, and so it will be convenient to use the $\operatorname{SL}(2, R)$-covariant basis rather than the one that transforms under the nonlinear $\mathrm{SO}(2)$. Similarly, the operators that appear in the general superalgebra (4) must be allowed to depend on the moduli and be consistent with the new basis. Hence in Eq. (4) one has $p_{\mu}^{i}(\kappa), x_{\mu_{1} \cdots \mu_{5}}^{i}(\kappa)$, and $y_{\mu_{1} \mu_{2} \mu_{3}}(\kappa)$, which are in triplets and singlets of the linear $\mathrm{SL}(2, R)$.

We will now determine the moduli $\kappa$ on the basis of their $\operatorname{SL}(2, R)$ properties. They are Lorentz invariant in ten dimensions, but they transform nontrivially $\kappa \rightarrow \kappa_{g}$ under $g \in$ $\mathrm{SL}(2, R)$. In particular $\tau \rightarrow \tau_{g}$ must be given by Eq. (6). In the new basis $\operatorname{SL}(2, R)_{U}$ transformations are equivalent to applying $\mathrm{SL}(2, R)_{B}$ transformations [instead of $\mathrm{SO}(2)$ ] on the doublet indices $a$ on $Q_{\alpha a}(\kappa)$ or triplet indices $i$ on $p_{\mu}^{i}(\kappa)$, $x_{\mu_{1} \cdots \mu_{5}}^{i}(\kappa)$. That is,

$$
\begin{gathered}
Q_{\alpha a}(\kappa) \rightarrow Q_{\alpha a}\left(\kappa_{g}\right)=g_{a}^{b} Q_{\alpha b}(\kappa), \\
p_{\mu}^{i}(\kappa) \rightarrow p_{\mu}^{i}\left(\kappa_{g}\right)=T_{j}^{i}(g) p_{\mu}^{j}(\kappa), \text { etc. },
\end{gathered}
$$

where $T_{j}^{i}(g)$ is the triplet representation of $g \in \mathrm{SL}(2, R)$. Under these transformations we must seek a moduliindependent momentum operator $P_{\mu}$ that is a singlet of $\operatorname{SL}(2, R)_{U=B}$. Furthermore, one knows that the $\operatorname{SL}(2, R)_{U}$ doublet of supergravity fields $B_{\mu \nu}^{a}$ must couple to a doublet of strings, the so-called $(p, q)$ strings [10]. So we must also seek a doublet of vectors $W_{\mu}^{a}=\left(E_{\mu}, D_{\mu}\right)$ representing the elementary string [Neveu-Schwarz-Neveu-Schwarz (NSNS) sector] and the $D$-1-string [Ramond-Ramond (R-R) sector]. The three vectors $p_{\mu}^{i}(\kappa)$ that appear in the superalgebra must be a combination of the moduli-independent singlet and doublet vectors $P_{\mu}, W_{\mu}^{a}$ with coefficients that depend on the moduli $\kappa$. Hence we must have

$$
p_{\mu}^{i}(\kappa)=P_{\mu} v^{i}(\kappa)+\varepsilon_{a b} W_{\mu}^{a} \lambda^{i b}(\kappa),
$$

where we have used the Levi-Cività symbol $\varepsilon_{a b}$ to combine doublet indices into a singlet. We have introduced $3+3 \times 2$ $=9$ functions $v^{i}(\kappa), \lambda^{i b}(\kappa)$, but to define the normalizations of $P_{\mu}, W_{\mu}^{a}$ we must normalize the functions correctly. Thus we must find $9-3=6$ constructed from moduli. Consistency of the transformation properties (10) demands that these functions satisfy

$$
v^{i}\left(\kappa_{g}\right)=T_{j}^{i}(g) v^{j}(\kappa), \quad \lambda^{i a}\left(\kappa_{g}\right)=T_{j}^{i}(g) g_{b}^{a} \lambda^{j b}(\kappa) .
$$

Then $P_{\mu}$ is a singlet and $W_{\mu}^{a}$ is a doublet as desired:

$$
P_{\mu} \rightarrow P_{\mu}, \quad W_{\mu}^{a} \rightarrow g_{b}^{a} W_{\mu}^{b} .
$$

It is convenient to rewrite the functions $v^{i}(\kappa), \lambda^{i b}(\kappa)$ by converting the vector index $i$ into a pair of symmetrized spinor indices; therefore, we define the nine functions $G_{a b}, \theta_{a b}^{c}$ :

$$
G_{a b}(\kappa) \equiv\left(i \tau_{2} \tau_{i}\right)_{a b} v^{i}(\kappa), \quad \theta_{a b}^{c}(\kappa) \equiv\left(i \tau_{2} \tau_{i}\right)_{a b} \lambda^{i c}(\kappa),
$$

where the $a b$ indices are symmetric. The transformation laws (12) are equivalent to

$$
\begin{gathered}
G_{a b}\left(\kappa_{g}\right)=\left(g G(\kappa) g^{T}\right)_{a b}, \\
\theta_{a b}^{c}\left(\kappa_{g}\right)=g_{d}^{c}\left(g \theta^{d}(\kappa) g^{T}\right)_{a b} .
\end{gathered}
$$

In terms of these the general superalgebra (2) and (4) takes the form

$$
\begin{aligned}
\left\{Q_{\alpha a}, Q_{\beta b}\right\}= & \gamma_{\alpha \beta}^{\mu}\left(P_{\mu} G_{a b}+\varepsilon_{a b} W_{\mu}^{a} \theta_{a b}^{c}\right) \\
& +\gamma_{\alpha \beta}^{\mu_{1} \mu_{2} \cdots \mu_{5}}\left(\begin{array}{c}
Y_{\mu_{1} \cdots \mu_{5}} \widetilde{G}_{a b} \\
+\varepsilon_{d c} X_{\mu_{1} \cdots \mu_{5}}^{d} \widetilde{\theta}_{a b}^{c}
\end{array}\right) \\
& +\gamma_{\alpha \beta}^{\mu_{1} \mu_{2} \mu_{3}} T_{\mu_{1} \mu_{2} \mu_{3}} \varepsilon_{a b} N(\kappa),
\end{aligned}
$$

where, like the one-branes, we have rewritten the three selfdual five-branes $x_{\mu_{1} \cdots \mu_{5}}^{i}$ in terms of a singlet $Y_{\mu_{1} \cdots \mu_{5}}$ and a doublet $X_{\mu_{1} \cdots \mu_{5}}^{d}$ by introducing nine functions $\widetilde{G}, \widetilde{\theta}$ that have identical transformation properties to those of $G, \theta$. We have also introduced an overall normalization function $N(\kappa)$ in front of the three-brane $T_{\mu_{1} \mu_{2} \mu_{3}}$. This function is a scalar under $\operatorname{SL}(2, R)$ transformations $N(\kappa) \rightarrow N\left(\kappa_{g}\right)$. We have taken the pairs $G, \theta$ and $\widetilde{G}, \widetilde{\theta}$ to be different in general, but they may be the same depending on the details of the theory. Similarly $N(\kappa)$ may be just a constant.

In this way we have shifted all the moduli dependence on the coefficients $G, \theta, \widetilde{G}, \widetilde{\theta}, N$ and identified the various operators that are independent of the moduli. When $\operatorname{SL}(2, R)_{U}$ transformations are applied $\kappa \rightarrow \kappa_{g}$ it induces $\kappa$-independent $\operatorname{SL}(2, R)_{B=U}$ transformations on the operators, such that $\left(P_{\mu}, Y_{\mu_{1} \cdots \mu_{5}}, T_{\mu_{1} \mu_{2} \mu_{3}}\right)$ behave as singlets and $\left(W_{\mu}^{a}, X_{\mu_{1} \cdots \mu_{5}}^{a}\right)$ behave as doublets. 


\section{Functions}

Now, what are the functions that have these properties? We will construct $G, \theta$, and assume that in the simplest case $\widetilde{G}, \widetilde{\theta}$ are the same except possibly for overall factors similar to $N$. Introduce two $2 \times 2$ real matrices $A_{a a^{\prime}}(\kappa)$ and $B_{a a^{\prime}}(\kappa)$ with $\operatorname{determinants} \operatorname{det} A=1, \operatorname{det} B=1$, and postulate that the moduli $\kappa$ transform such that these matrices transform only on the left side when $\kappa$ is replaced by $\kappa_{g}$ :

$$
A\left(\kappa_{g}\right)=g A(\kappa), \quad B\left(\kappa_{g}\right)=g B(\kappa) .
$$

Recall that after taking into account normalizations, taken together $G_{a b}(\kappa)$ and $\theta_{a b}^{c}(\kappa)$ contain six independent functions of the moduli. On the other hand, $A, B$ taken together also have six independent functions of the moduli, and so we may expect to construct the most general $G_{a b}(\kappa)$ and $\theta_{a b}^{c}(\kappa)$ in terms of $A(\kappa)$ and $B(\kappa)$. We can now give the nine functions that have the desired transformation properties:

$$
\begin{gathered}
G_{a b}(\kappa)=\left(A 1 A^{T}\right)_{a b}, \\
\theta_{a b}^{c}(\kappa)=\left(A \tau^{1} A^{T}\right)_{a b} B_{c 1}+\left(A \tau^{3} A^{T}\right)_{a b} B_{c 2} .
\end{gathered}
$$

Next we construct $A, B$ for the general case. Each $2 \times 2$ real matrix $A, B$ that transforms only on the left as in Eq. (17) is really equivalent to the vector representation $(1 / 2,1 / 2)$ of $\operatorname{SL}(2, R)_{B} \otimes \operatorname{SL}(2, R)_{B^{\prime}}=\mathrm{SO}(2,2)$, where $\operatorname{SL}(2, R)_{B^{\prime}}$ is not activated by the $\operatorname{SL}(2, R)_{B=U}$ transformation of the moduli $\kappa \rightarrow \kappa_{g}$ as seen in Eq. (17). Therefore $A, B$ can be parametrized by two "on-shell" vectors $u^{m}, p^{m}$ of $\mathrm{SO}(2,2)$ by expanding each matrix in a basis of Pauli matrices $\tau_{m}$ $=\left(\tau_{1}, i \tau_{2}, \tau_{3}, 1\right):$ i.e.,

$$
B_{a b^{\prime}}=p^{m}\left(\tau_{m}\right)_{a b^{\prime}}, \quad \operatorname{det} B=p^{m} p^{n} \eta_{m n}=1,
$$

where the signature $\eta_{m n}=(+,-,+,-)$. Similar expressions hold for $A(u)$. The moduli $u^{m}$ and $p^{m}$ are generally independent, but they could be related to each other in special corners of the theory. In particular, in the supergravity limit they are probably related since there is only a single $\tau$ modulus in that case. In this paper we will show that for computations in BPS sectors $A(u)$ disappears, and so we will not need to know any of the possible relations between the $u^{m}$ and $p^{m}$ moduli. Furthermore, for BPS sectors, only one combination of the $p^{m}$ corresponding to the $\tau$ will survive, but the remaining components must appear in more general computations. We will next see how the $\tau$ modulus is embedded in $\operatorname{SO}(2,2)$.

Following the ideas of $S$ theory [6] it is tempting to interpret the index $m$ as corresponding to two timelike and two spacelike dimensions beyond the 10D. Hence, we will label $m=11,12,13,14$. The explicit matrices are expressed in terms of the light-cone-type combinations of the components of $p^{m}$ :

$$
B=\left(\begin{array}{ll}
p^{14}+p^{13} & p^{11}+p^{12} \\
p^{11}-p^{12} & p^{14}-p^{13}
\end{array}\right)
$$

According to $\operatorname{SL}(2, R)_{U}$ transformations of Eq. (17) each column of this matrix transforms as a doublet. The same holds for the two columns of $A(u)$. We may now define the $\tau$ modulus in terms of these. Define a complex doublet by combining the two columns of $B$ :

$$
\left(\begin{array}{l}
z_{1} \\
z_{2}
\end{array}\right)=\left(\begin{array}{l}
\left(p^{14}+p^{13}\right)-i\left(p^{11}+p^{12}\right) \\
\left(p^{11}-p^{12}\right)-i\left(p^{14}-p^{13}\right)
\end{array}\right) .
$$

The ratio $\tau \equiv z_{1} / z_{2}$ undergoes the Möbius transformation given in Eq. (6). Note that the determinant (or "on-shell") condition for $B$ is equivalent to $\operatorname{Im}\left(z_{1} z_{2}^{*}\right)=1$ or to $\operatorname{Im}(\tau)$ $=\left|z_{2}\right|^{-2}$, which requires $\tau$ to be in the upper half plane. Thus, the complex modulus $\tau$ plus the phase of $z_{2}$ are three moduli equivalent to the matrix $B$. We can write

$$
\left(\begin{array}{l}
z_{1} \\
z_{2}
\end{array}\right)=\frac{e^{i \phi}}{\sqrt{\operatorname{Im} \tau}}\left(\begin{array}{l}
\tau \\
1
\end{array}\right)
$$

and

$$
B(\tau, \phi)=\left(\begin{array}{ll}
\sqrt{\operatorname{Im} \tau} & \frac{\operatorname{Re} \tau}{\sqrt{\operatorname{Im} \tau}} \\
0 & \frac{1}{\sqrt{\operatorname{Im} \tau}}
\end{array}\right)\left(\begin{array}{cc}
-\sin \phi & -\cos \phi \\
\cos \phi & -\sin \phi
\end{array}\right) .
$$

Thus, the moduli correspond to an $\mathrm{SO}(2,2)$ vector $p^{m}$ that takes various values as a function of the coupling constant $g_{s}$ and other moduli, while maintaining the $\mathrm{SO}(2,2)$ "'on-shell", constraint $p \cdot p=1$.

A similar statement can be made about $A$. From the structure of Eq. (18) it is evident that $A$ corresponds to an $\operatorname{SL}(2, R)$ freedom for changing the basis labeled by $a$ in $Q_{\alpha a}(\kappa)$. This is related to the freedom of choosing the basis, as discussed in the beginning of Sec. II B. This freedom can be used to relate the $u^{m}$ and $p^{m}$ moduli or keep them independent depending on the particular corner of the theory that one may wish to explore. For example, one may relate the heterotic sector to the type-IIB theory by adjusting the $u^{m}$ moduli relative to $p^{m}$ moduli and taking appropriate limits so that the heterotic superalgebra emerges from the type-IIB superalgebra discussed above.

For general values of the moduli $(\tau, \phi)$ we have obtained the nonperturbative form of the superalgebra (16). In addition we have gained the following perspective: The $\operatorname{SL}(2, R)_{U=B}$ transformations are a subset of Lorentz transformations in the $(2,2)$ space of the extra dimensions. Lorentz transformations are equivalent to making the $\kappa \rightarrow \kappa_{g}$ transformations on the moduli, including

$$
e^{i \phi_{g}}=e^{i \phi} \frac{(c \tau+d)}{|(c \tau+d)|},
$$

in addition to Eq. (6), and similarly for the moduli that parametrize $A$. Rewriting the moduli in terms of the components of the $\mathrm{SO}(2,2)$ vectors brings out the 14D structure of the theory

$$
\begin{gathered}
\tau=\frac{\left(p^{14}+p^{13}\right)-i\left(p^{12}+p^{11}\right)}{\left(p^{12}-p^{11}\right)-i\left(p^{14}-p^{13}\right)}, \\
\tan \phi=\left(p^{14}-p^{13}\right) /\left(p^{12}-p^{11}\right) .
\end{gathered}
$$


Up to now we have referred to continuous transformations $g \in \mathrm{SL}(2, R)$, such as those that occur in Eqs. (6) and (15). Recall that $g$ also acts on the doublet $W_{\mu}^{a}$. These operators have discrete eigenvalues, (the winding numbers) in the compactified dimensions. Since the SL(2) transformation acts on a space of quantized eigenvalues, only the discrete subgroup $\operatorname{SL}(2, Z)$ remains as a symmetry in the compactified theory.

It is worth asking the question of whether our construction has any relation to $F$-theory [9]. We do not know the answer, but one should note that instead of defining a torus (which involved a worrisome analytic continuation in $F$ theory), we have introduced $\mathrm{SO}(2,2)$ and noticed four hidden dimensions rather than two. It may be interesting to review $F$ theory constructions to see if they can be reinterpreted in terms of a 14-dimensional formalism.

\section{NONPERTURBATIVE COMPUTATIONS}

Certain properties of nonperturbative BPS states can be computed algebraically by using the property that a BPS state corresponds to a short representation of the superalgebra. In a short representation a subset of supergenerators must vanish. If the superalgebra has the form

$$
\left\{Q_{\alpha a}, Q_{\beta b}\right\}=S_{\alpha a, \beta b},
$$

then in a short representation $S_{\alpha a, \beta b}$ must have zero eigenvalues. This implies that the determinant is zero

$$
\operatorname{det}\left(S_{\alpha a, \beta b}\right)=0
$$

This is a constraint that involves the momentum, the various bosonic charges, and the moduli. It must hold at any coupling since it is a purely algebraic property. Hence all solutions of the determinant equation correspond to nonperturbative results in the theory.

The determinant is invariant under similarity transformations. If $\widetilde{G}, \widetilde{\theta}$ are assumed to be proportional to $G, \theta$, then the moduli $A(u)$ can be removed by a similarity transformation consisting of the $32 \times 32$ matrix $1_{16} \otimes A(u)$. Hence the moduli $u^{m}$ do not contribute in the BPS sector, and the matrix $A$ that appears in Eq. (18) can be effectively gauge fixed to $A=1$ in this sector.

In addition, one can make further orthogonal transformations on $S_{\alpha a, \beta b}$ of the form $1_{16} \otimes T_{2}$ which do not change the gauge-fixed value of $A=1$, since $T_{2} 1 T_{2}^{T}=1$. These are given by $T_{2}=\exp \left(i \tau^{2} \alpha\right)$, and they mix $\tau^{1}$ with $\tau^{3}$. Then $\alpha$ can be chosen to remove the modulus $\phi$ from the expressions in Eq. (18) provided we are in the BPS sector. There remains only the complex modulus $\tau$ as the only gauge-independent parameter in the BPS sector.

These last two paragraphs explain why $\tau$ appears as the only nonperturbative parameter in the following BPS calculations, although we will include the most general form, including $\phi, A(u)$, without choosing the gauges above. In nonBPS sectors one does not have the gauge freedom described above, and so at least the $\phi$ modulus must play a role in the general sector. In addition the $u^{m}$ moduli are probably important in general. Understanding their role may shed light on 14 dimensions.

\section{A. Tension of $\left(q_{1}, q_{2}\right)$ strings}

The $\left(q_{1}, q_{2}\right)$ strings are strings that carry $q_{1}$ units of NS-NS winding numbers and $q_{2}$ units of R-R winding numbers, for $q_{1}, q_{2}$ relatively prime integers. They occur as BPS states when the ninth dimension is compactified on a circle of radius $R_{9}$. To compute the string tension we set to zero all five-branes $Y_{\mu_{1} \cdots \mu_{5}}, X_{\mu_{1} \cdots \mu_{5}}^{a}=0$ and three-branes $T_{\mu_{1} \mu_{2} \mu_{3}}$ $=0$, and keep only the Kaluza-Klein momentum $P_{9}$ $=n / R_{9}$, and the ninth components of the two strings $W_{9}^{a}$ $=m^{a} R_{9}$ which represent the winding numbers $m^{a}$ $=\left(m_{1}, m_{2}\right)$ of the NS-NS and R-R strings (or elementary string and $D$-1-string). The momentum in nine dimensions, $P_{\mu}$, is also present, but using the 9D Lorentz symmetry, the momentum can be taken at rest $P_{\mu}=(M, \sigma)$. On such states the superalgebra takes the form

$$
S_{\alpha a, \beta b}=\gamma_{\alpha \beta}^{0} M G_{a b}+\gamma_{\alpha \beta}^{9}\left(P_{9} G_{a b}+W_{9}^{1} \theta_{a b}^{2}-W_{9}^{2} \theta_{a b}^{1}\right),
$$

where we insert the general expressions of Eq. (18). Recall that $\gamma^{0}=1_{16}$ in the Weyl sector. For convenience we may choose a diagonal $\gamma^{9}=\sigma_{3} \otimes 1_{8}$. In computing the determinant, $A(u)$ drops out since it has the form of a similarity transformation. Then one can replace $G \rightarrow 1, \theta^{2} \rightarrow B^{21} \tau_{1}$ $+B^{22} \tau_{3}$, and $\theta^{1} \rightarrow B^{11} \tau_{1}+B^{12} \tau_{3}$ and compute the determinant easily:

$$
\begin{aligned}
\operatorname{det}= & {\left[\left(M+P_{9}\right)^{2}-W_{9}^{a}\left(B^{-1 T} B^{-1}\right)_{a b} W_{9}^{b}\right]^{8} } \\
& \times\left[\left(M-P_{9}\right)^{2}-W_{9}^{a}\left(B^{-1 T} B^{-1}\right)_{a b} W_{9}^{b}\right]^{8} .
\end{aligned}
$$

For generic values of $P_{9}, W_{9}^{a}$ there are four distinct eigenvalues where the determinant vanishes. The BPS mass corresponds to the largest eigenvalue, because the anticommutator of each of the 32 supercharges must be either positive or zero. Thus,

$$
M_{\mathrm{BPS}}=\frac{|n|}{R_{9}}+R_{9} \sqrt{m^{a} m^{b} g_{a b}(\tau)},
$$

where

$$
\begin{gathered}
g_{a b}(\tau)=\left(B^{-1 T} B^{-1}\right)_{a b}, \\
=\frac{1}{\operatorname{Im} \tau}\left(\begin{array}{cc}
1 & -\operatorname{Re} \tau \\
-\operatorname{Re} \tau & |\tau|^{2}
\end{array}\right) .
\end{gathered}
$$

For generic $n, m^{a}$, the multiplicity of the zero is 8 , and therefore eight supercharges vanish, giving a BPS space of strings with $1 / 4$ supersymmetry. In general the integers $\left(m_{1}, m_{2}\right)$ are not relatively prime. Factoring out the largest common factor

$$
\left(m_{1}, m_{2}\right)=m\left(q_{1}, q_{2}\right),
$$

one obtains

$$
\begin{gathered}
M_{\mathrm{BPS}}=\frac{|n|}{R_{9}}+R_{9}|m| T_{q_{1}, q_{2}}, \\
T_{q_{1}, q_{2}}(\tau)=\sqrt{q^{a} q^{b} g_{a b}(\tau)}
\end{gathered}
$$


where $q_{1}, q_{2}$ are relatively prime. $T_{q_{1}, q_{2}}$ is interpreted as the tension of the $\left(q_{1}, q_{2}\right)$ string at any coupling $\tau$ in agreement with [10]. In particular the NS-NS string $q^{a}=(1,0)$ and R-R string $q^{a}=(0,1)$ have tensions

$$
T_{1,0}=\frac{1}{\sqrt{\operatorname{Im} \tau}}, \quad T_{0,1}=\frac{|\tau|}{\sqrt{\operatorname{Im} \tau}} .
$$

By comparing to Eq. (8) we see that in the weak coupling limit $\sqrt{\operatorname{Im} \tau} \rightarrow g_{s}^{-1}$, so that the mass of the R-R string $q^{a}$ $=(0,1)$ goes to infinity and decouples from the perturbative theory. By contrast, at strong coupling, the NS-NS string $q^{a}=(1,0)$ becomes infinitely massive and decouples. These two limits are interchanged by $S$ duality, which is an element of $\operatorname{SL}(2, Z)$.

Under $\operatorname{SL}(2, Z)$ the quanta $m^{a}$ transform as a doublet and $\tau$ transforms under Möbius transformations as in Eq. (6), while the mass formula or the tension remains invariant. This invariance was built in the superalgebra, and so it is no surprise that it is present in the mass formula derived through an SL $(2, Z)$ covariant procedure. By obtaining this well-known result from the nonperturbative superalgebra we have illustrated that the algebra encodes useful information.

\section{B. Mass and entropy of BPS extremal black holes}

Consider supergravity as the low energy limit of any typeIIB theory (superstring, $M$-theory, etc.). It is known that in supergravity there are black hole solutions. At space infinity, far away from the black hole, the field configurations for these solutions are proportional to $p$-brane charges. These charges that define the black hole state are the ones that appear in the superalgebra as central extensions. It is known that the extremal black holes are BPS states, and therefore many of their properties, such as masses, can be determined by algebraic means. In particular, it is known that the area of the horizon of the extremal black hole, which is proportional to the entropy, is given as a $U$-duality-invariant expression constructed from the central extensions of the superalgebra [11]. We can therefore apply a purely algebraic approach to compute the mass and entropy of supersymmetric extremal black holes. With our formalism we can compute these quantities at any value of the coupling.

As an example we consider a popular black hole configuration in five dimensions that is constructed from $Q_{1}$ $D$-1-branes and $Q_{5} D$-5-branes. It has the following three charges [14]: Kaluza-Klein momentum $P_{9}=n / R_{9}$, string winding $W_{9}^{(2)}=Q_{1} R_{9}$, and $D$-5-brane winding $X_{56789}^{(2)}$ $=Q_{5} R_{9} V_{4}$. Here $n, Q_{1}, Q_{5}$ are quantized integers of any sign (brane, antibrane, etc.), $R_{9}$ is the radius of the circle for the ninth compactified dimension, and $R_{9} V_{4}$ is the volume of the $D$-5-brane. There is also a momentum in five-dimensions which is taken at rest $P_{\mu}=(M, \overrightarrow{0})$, with $M$ positive. All other extensions in the superalgebra are set to zero for this black hole state. The nonperturbative superalgebra takes the form

$$
\begin{aligned}
S_{\alpha a, \beta b}= & \left(\gamma_{\alpha \beta}^{0} M+\gamma_{\alpha \beta}^{9} P_{9}\right) G_{a b}-\gamma_{\alpha \beta}^{9} W_{9}^{(2)} \theta_{a b}^{(1)} \\
& -\gamma_{\alpha \beta}^{56789} X_{56789}^{(2)} \theta_{a b}^{(1)}
\end{aligned}
$$

As in the previous subsection $A$ drops out in the computation of the determinant, and so we may replace $G \rightarrow 1$, $\theta^{1} \rightarrow B^{11} \tau_{1}+B^{12} \tau_{3}$. By making similarity transformations which do not change the determinant of $S$, one can choose a new basis for the $\tau$ matrices such that $\theta^{1}$ is diagonalized in the new form

$$
\begin{aligned}
\theta^{1} & \rightarrow \tau_{3} \sqrt{\left(B^{11}\right)^{2}+\left(B^{12}\right)^{2}} \\
& =\tau_{3} \frac{|\tau|}{\sqrt{\operatorname{Im} \tau}} .
\end{aligned}
$$

Recall that in the Weyl basis $\gamma^{0}=1_{16}$, furthermore, $\gamma^{9}$ and $\gamma^{56789}$ commute with each other. So one may use the following $16 \times 16$ diagonal matrices to represent them:

$$
\gamma^{9}=1_{4} \otimes \sigma_{3} \otimes 1_{2}, \quad \gamma^{56789}=1_{4} \otimes 1_{2} \otimes \sigma_{3} .
$$

In this form the $32 \times 32$ matrix $S_{\alpha a, \beta b}$ is diagonal. For generic $M, P_{9}, W_{9}^{(2)}$, and $X_{56789}^{(2)}$ it has eight distinct eigenvalues, each one being fourfold degenerate, indicating that this configuration has four zero supercharges if the mass $M$ is chosen equal to the BPS mass. The relative signs must be chosen so that the BPS mass is the largest eigenvalue, so that each of the 32 supercharges has a positive anticommutator. Therefore we obtain only positive contributions from each term:

$$
\begin{aligned}
M_{\mathrm{BPS}} & =\left|P_{9}\right|+\left(\left|W_{9}^{(2)}\right|+\left|X_{56789}^{(2)}\right|\right) \frac{|\tau|}{\sqrt{\operatorname{Im} \tau}} \\
& =\frac{|n|}{R_{9}}+\left(\left|Q_{1}\right|+V_{4}\left|Q_{5}\right|\right) \frac{R_{9}|\tau|}{\sqrt{\operatorname{Im} \tau}} .
\end{aligned}
$$

Since the zero eigenvalue of $S_{\alpha a, \beta b}$ is fourfold, four supercharges vanish. Therefore this BPS spectrum of black holes has $1 / 8 \quad(=4 / 32)$ supersymmetry at generic values of $n, Q_{1}, Q_{5}$.

This nonperturbative result, valid for all values of the coupling constant and moduli, is more general than others available in the literature. It is in agreement with [14] for the special values $\operatorname{Re} \tau=0$ and $\sqrt{\operatorname{Im} \tau} \sim 1 / g_{s}$ valid at weak coupling. As demonstrated, it follows only from algebraic properties of the superalgebra, plus physical insight into the meaning of the various parameters that characterize the quantum numbers of the black hole. Note that the nonperturbative tension of the $D$-1-brane is consistent with the previous subsection.

The entropy of an extremal black hole in five dimensions is expressed generally by $S=A_{5} / 4 G_{5}=2 \pi \sqrt{\left|I_{5}\right|}$, where $G_{5}$ is the Newton constant in five dimensions and $A_{5}$ is the area of the black hole. This expression is given by the $E_{6,6}$ cubic invariant $I_{5}$ constructed from the central extensions [11-13]

$$
I_{5}=C \operatorname{Tr}(Z \Omega Z \Omega Z \Omega)
$$

where $\Omega$ is the $\operatorname{Sp}(8)$-invariant metric and $Z$ contains the 27 of $E_{6,6}$ in the form of an $8 \times 8$ antisymmetric matrix of central extensions which satisfies $\operatorname{Tr}(Z \Omega)=0$. The factor $C$ in front accounts for a renormalization of the central extensions, and it will be explained below. In our case the form of 
the central extension $Z$, in ten-dimensional units, is given by the matrix in Eq. (35) excluding the mass term. An $8 \times 8$ antisymmetric $Z$ is formed by using the $\gamma$ matrices in Eq. (37), excluding the first factor $1_{4}$ (spinor space for the five dimensions) and replacing the diagonal $\sigma_{3}$ 's by antisymmetric $\sigma_{2}$ 's:

$$
\begin{aligned}
Z= & \sigma_{2} \otimes 1_{2} \otimes G P_{9}-\sigma_{2} \otimes 1_{2} \otimes \theta^{(1)} W_{9}^{(2)}-1_{2} \otimes \sigma_{2} \\
& \otimes \theta^{(1)} X_{56789}^{(2)} .
\end{aligned}
$$

The $8 \times 8$ antisymmetric $\Omega$ orthogonal to $Z$ is $\Omega=1_{2} \otimes \sigma_{2}$ $\otimes G^{-1}$. Then $I_{5}$ reduces to

$$
I_{5}=24 C \operatorname{Tr}\left(\theta^{(1)} G^{-1} \theta^{(1)} G^{-1}\right) P_{9} W_{9}^{(2)} X_{56789}^{(2)} .
$$

This expression is simplified by using the property of the trace to eliminate the matrix $A$, thus substituting again $G \rightarrow 1_{2}$ and $\theta^{1} \rightarrow B^{11} \tau_{1}+B^{12} \tau_{3}$. The result is

$$
I_{5}=24 C \frac{2|\tau|^{2}}{\operatorname{Im} \tau} R_{9} V_{4} n Q_{1} Q_{5}=n Q_{1} Q_{5},
$$

which gives the well-known moduli-independent entropy [14], provided

$$
C=\frac{\operatorname{Im} \tau}{48 R_{9} V_{4}|\tau|^{2}} .
$$

The factor $C$ is nontrivial because we have expressed the central extensions in ten dimensional units, as they appear in the 10D superalgebra, which is renormalized relative to the one in [11-13]. Renormalization factors for the metric and fermions in 5D introduce a renormalization of the 5D supercharges and, hence, of the central extensions. We have found that $C$ has nonperturbative contributions.

We have demonstrated the usefulness of the approach by deriving new nonperturbative results for the mass $M_{\mathrm{BPS}}$ and for the factor $C$ related to renormalization.

Many more new nonperturbative results can be derived for other physical states by following a similar procedure for any $p$-brane configuration. Furthermore, since the superalgebra must be valid for matrix elements of scattering states, there must be various sum rules and relations among scattering amplitudes that can be derived algebraically by sandwiching the superalgebra between scattering states. The methods for obtaining such results are similar to the current algebra techniques used in strong and weak interactions in the sixties. Such results will be valid nonperturbatively for any value of the coupling constant since the superalgebra is nonperturbative. It would be interesting to derive such relations in the future.

\section{IV. $S$ THEORY BASED ON MATRICES IN 14D}

Some very complicated arguments that yield nonperturbative results about the theory seem to rely on dynamics, but the results shown above, and many others, really depend on the properties of the superalgebra and would be the same in any model with the same extended supersymmetry. As advocated in the $S$-theory approach [4], it is useful to recognize the general properties of the symmetry and distinguish the results that follow from it independently from the dynamics. Of course, dynamical realizations of the superalgebra provide greater insight into the physics. Using the general algebraic properties as a guide to construct the dynamics is bound to be fruitful. We will follow this route to suggest an outline for a dynamical model below.

We sketch the basic elements of a new dynamical matrix construction [15] that realizes the bigger picture compatible with the general superalgebra. Although the emphasis in this paper is not on this theory, a brief outline is included here in order to provide a perspective for a more concrete realization of the algebraic properties of $S$ theory discussed in this paper and elsewhere $[4,6]$.

Let us first recall the general algebraic picture that goes beyond the structures in the previous sections. It was claimed in [6] that the unified theory that exhibits explicitly at least ten-dimensional Lorentz covariance and type-IIA or type-IIB duality is naturally a 14-dimensional theory with signature $(11,3)$, because it must be formulated in terms of 64 real supercharges. Supersymmetry in higher than 11 dimensions deviates substantially from the usual form. In 14D the general superalgebra, with 64 real supercharges corresponding to the Weyl-Majorana spinor, is given by

$$
\left\{Q_{\alpha}, Q_{\beta}\right\}=\gamma_{\alpha \beta}^{\mu_{1} \mu_{2} \mu_{3}} T_{\mu_{1} \mu_{2} \mu_{3}}+\gamma_{\alpha \beta}^{\mu_{1} \cdots \mu_{7}} Z_{\mu_{1} \cdots \mu_{7}}^{+},
$$

where $Z_{\mu_{1} \cdots \mu_{7}}^{+}$is self dual. As discussed in [6], for the theory to have effectively only 32 real supercharges, a gauge symmetry must be built in such that it provides the constraint that this supersymmetry must live only in the short multiplet sectors. A fully $\mathrm{SO}(11,3)$-covariant equation that ensures this property is the master equation (that should follow from a gauge invariance)

$$
\operatorname{det}\left(\gamma^{\mu_{1} \mu_{2} \mu_{3}} T_{\mu_{1} \mu_{2} \mu_{3}}+\gamma^{\mu_{1} \cdots \mu_{7}} Z_{\mu_{1} \cdots \mu_{7}}^{+}\right)=0 .
$$

As explained in [6], this BPS-like equation has four ${ }^{4}$ main branches of solutions falling into sectors $A, B, C$, and $D$ characterized by the maximal isometries of their superalgebras (if certain moduli are frozen; see [6]):

$$
\begin{gathered}
A: \mathrm{SO}(10,2) \otimes \mathrm{SO}(1,1), \\
B: \mathrm{SO}(9,1) \otimes \mathrm{SO}(2,2), \\
C: \mathrm{SO}(8,0) \otimes \mathrm{SO}(3,3), \\
D: \mathrm{SO}(6,2) \otimes \mathrm{SO}(3,1) \otimes \mathrm{SO}(1,1) .
\end{gathered}
$$

Each branch has 32 real supercharges, and contains subbranches with fewer supercharges. The branches labeled by $A$ and $B$, when viewed from the point of view of ten dimensions, have precisely the maximally extended superalgebras of types IIA, and IIB in ten dimensions. The $C$ and $D$ branches do not have 10D covariance but have 4D covariance since they contain $\mathrm{SO}(3,1)$ as a subgroup. Subbranches with less supersymmetry, corresponding to heterotic, type-I,

\footnotetext{
${ }^{4}$ In [6] the fourth branch was missed. I thank S. Yankielowicz for pointing it out.
} 
and all compactifications, are all solutions of the same 14D master equation. The symmetries of the master equation (similarity transformations on the $64 \times 64$ matrix in spinor space) permit transformations from one solution to another, and these correspond to dualities [6].

Now we present some initial ideas for building a dynamical model compatible with the general algebraic picture. It is based on an $\mathrm{SO}(11,3)$-covariant infinite dimensional matrix model that borrows from the current ideas in matrix models for $M$ theory [7]. Suppose there is an action for a matrix model in 14 dimensions, with 14 bosonic Hermitian matrices $X_{i j}^{\mu}, \mu=1, \ldots, 14$, with signature $(11,3)$, and 64 fermionic Hermitian matrices $\theta_{i j}^{\alpha}, \alpha=1, \ldots, 64$, in the Weyl-Majorana spinor representation of $\mathrm{SO}(11,3)$. Since supersymmetry in higher than 11 dimensions deviates substantially from the usual form, as seen in Eq. (44), the 14D matrix action cannot closely resemble the reduced super-Yang-Mills action in ten dimensions. However, the superalgebra constructed from the matrices is expected to have extensions that are similar to the ones found in 10D matrix models. Namely, consider the form

$$
\begin{aligned}
\left\{Q_{\alpha}, Q_{\beta}\right\}= & \gamma_{\alpha \beta}^{\mu_{1} \mu_{2} \mu_{3}}\left[\frac{1}{N} \operatorname{Tr}\left(X_{\mu_{1}} X_{\mu_{2}} X_{\mu_{3}}\right)+\cdots\right] \\
& +\gamma_{\alpha \beta}^{\mu_{1} \cdots \mu_{7}}\left[\frac{1}{N} \operatorname{Tr}\left(X_{\mu_{1}} X_{\mu_{2}} \cdots X_{\mu_{7}}\right)+\cdots\right],
\end{aligned}
$$

where the extensions constructed from matrices

$$
T_{\mu_{1} \mu_{2} \mu_{3}}=\frac{1}{2 N} \operatorname{Tr}\left(X_{\mu_{1}} X_{\mu_{2}} X_{\mu_{3}}-X_{\mu_{2}} X_{\mu_{1}} X_{\mu_{3}}\right)+\cdots,
$$

and similarly for $Z_{\mu_{1} \cdots \mu_{7}}^{+}$, are completely antisymmetric in the 14D Lorentz indices.

By now it is known that infinite dimensional matrices can be chosen to correspond to any collection of $p$-branes, with several types of $p$-branes appearing simultaneously. In our case we require that their configurations obey the master equation described above.

It is evident that all known $p$-brane solutions in 10D matrix models [7] form a subset of solutions to our equations since they can be embedded in 14D as in [6]. For example, one can take ten matrices as in [7] and four other constant matrices that point along constant orthogonal vectors embedded in $\mathrm{SO}(2,2)$. The determinant condition Eq. (45) is a restriction on the constant vectors such that the solution for $T_{\mu_{1} \mu_{2} \mu_{3}}, Z_{\mu_{1} \cdots \mu_{7}}^{+}$falls into one of the branches or subbranches in Eq. (46).

This approach, which is compatible with other current ideas on matrix models, contains dynamics in a duality and Lorentz covariant formalism, while providing a concrete realization of the algebraic properties of $S$ theory described in this paper and elsewhere $[4,6]$. The bigger picture outlined here schematically will be explored elsewhere in greater detail [15].

\section{ACKNOWLEDGMENTS}

I would like to thank Costas Kounnas for very valuable conversations that provided hints for connecting the $\mathrm{SO}(2,2)$ dimensions to moduli. I also acknowledge useful conversations with S. Yankielowicz, J. Maldacena, S. Ferrara, P. Townsend, A. Tseytlin, M. Cvetic, and T. Banks. This research was partially supported by U.S. DOE Grant No. DEFG03-84ER40168.
[1] J. Polchinski, Phys. Rev. Lett. 75, 4724 (1995). See also, J. Polchinski, S. Chaudhuri, and C. V. Johnson "Notes on Dbranes,' hep-th/9602052.

[2] I. Bars and A. Hanson, Phys. Rev. D 14, 1744 (1976); W. A. Bardeen, I. Bars, A. Hanson, and R. Peccei, ibid. 13, 2364 (1976).

[3] I. Bars, Phys. Rev. D 54, 5203 (1996); in Frontiers in Quantum Field Theory, Proceedings of the Conference, Toyonaka, Japan, 1995, edited by H. I. Itoyoma et al. (World Scientific, Singapore, 1996), hep-th/9604200.

[4] I. Bars, Phys. Rev. D 55, 2373 (1997); in Second International Sakharov Conference on Physics, Proceedings, Moscow, Russia, 1996, edited by I. Dremin and A. Semikhatov (World Scientific, Singapore, 1997), p. 355, hep-th/9608061.

[5] C. Hull and P. Townsend, Nucl. Phys. B438, 109 (1995).

[6] I. Bars, Phys. Lett. B 403, 257 (1997).
[7] T. Banks, W. Fischler, S. Shenker, and L. Susskind, Phys. Rev. D 55, 5112 (1997).

[8] See, e.g., M. B. Green, J. H. Schwarz, and E. Witten, Superstring Theory (Cambridge University Press, Cambridge, England, 1987), Vol. 1.

[9] C. Vafa, Nucl. Phys. B469, 403 (1996).

[10] J. H. Schwarz, Phys. Lett. B 360, 13 (1995).

[11] S. Ferrara and R. Kallosh, Phys. Rev. D 54, 1514 (1996); 54, 1525 (1996).

[12] R. Dijkgraaf, E. Verlinde, and H. Verlinde, Nucl. Phys. B486, 77 (1977).

[13] M. Cvetic and C. Hull, Nucl. Phys. B480, 296 (1996).

[14] A. Strominger and C. Vafa, Phys. Lett. B 379, 99 (1996); J. Maldacena, "Black holes and D-branes," hep-th/9705078, and references therein.

[15] I. Bars, "A 14D Matrix Theory with Manifest Duality" (in preparation). 